\title{
Lung Ultrasound Predicts Clinical Severity of COVID-19 Pneumonia
}

Alisha Goel ${ }^{1}$, Rajarajan Ganesan ${ }^{2}$, Neeti Dogra ${ }^{3}$, Arun Sharma ${ }^{4}$, Mandeep Garg ${ }^{5}$, Narender Kaloria ${ }^{6}$, Vighnesh Ashok ${ }^{7}$, Karan Singla ${ }^{8}$, Nischitha Gowda ${ }^{9}$, Subhrashis G Niyogi ${ }^{10}$, Navdeep Kaur ${ }^{11}$, Ashish Bhalla ${ }^{12}$, Varun Mahajan ${ }^{13}$, Sujitha R Karri ${ }^{14}$, Goverdhan D Puri ${ }^{15}$

\begin{abstract}
Background: Lung ultrasound is an easily available bedside imaging modality that has good specificity for the diagnosis of coronavirus disease2019 (COVID-19). Limited evidence is available on its utility to predict clinical severity.

Materials and methods: In this prospective observational study, adult patients with COVID-19 pneumonia admitted to the intensive care unit (ICU) of a tertiary care hospital between June 2020 and August 2020 were included. Lung ultrasound was performed in at least four areas in each examination and video loops were scored by two independent observers. The statistical relationship was assessed between median lung ultrasound score, chest $\mathrm{X}$-ray score, $\mathrm{P} / \mathrm{F}$ ratio (ratio of the partial pressure of oxygen in arterial blood to the fraction of inspired oxygen), ROX index, SOFA score, ICU stay, and mortality.

Results: Fifty lung ultrasound examinations were performed in 29 patients of age $54.7 \pm 15.3$ years. P/F ratio was $<300 \mathrm{~mm} \mathrm{Hg}$ during $80 \%$ of the examinations. There was moderate correlation between median lung ultrasound score and both ROX index (Spearman's rho $=-0.543, p<$ $0.001)$ and $\mathrm{P} / \mathrm{F}$ ratio (rho $=-0.522, p<0.001$ ), while there was weak correlation between chest $\mathrm{X}$-ray score and $\mathrm{ROX}$ index $($ rho $=-0.0 .345, p=$ 0.019 ) and no correlation between chest X-ray score and P/F ratio. There was correlation between chest X-ray score and ICU stay (rho $=0.434$, $p=0.049$ ). There was no difference in any of the parameters between survivors and non-survivors. Moderate inter-observer agreement was present between the two observers.

Conclusion: Bedside lung ultrasound examination, even with a limited number of views, can reflect the clinical severity of COVID-19 pneumonia. Keywords: Coronavirus disease-2019, Lung ultrasound, P/F ratio, Pneumonia, ROX index.

Journal of Perioperative Echocardiography (2019): 10.5005/jp-journals-10034-1103
\end{abstract}

\section{INTRODUCTION}

Coronavirus disease-2019 (COVID-19), caused by the SARS-CoV-2 virus, is characterized by predominant damage to the lungs. Coronavirus disease-2019 pneumonia is proposed to be due to the destruction of type-2 epithelial cells by the virus, later progressing to diffuse alveolar damage and eventually, acute respiratory distress syndrome. The initial imaging findings of COVID-19 is the presence of bilateral and multi-lobar peripheral ground-glass opacities on chest computed tomography scans. ${ }^{2}$ Corresponding lesions on lung ultrasound have also been found in these patients. ${ }^{3}$

Normal lung ultrasound image consists of reverberation artifacts created by the pleura. The lung ultrasound findings in COVID-19 consist of thickened pleural line, comet-tail artifact or B lines, subpleural pulmonary consolidation, and air bronchogram. ${ }^{4}$ Lung ultrasound has been shown to have higher sensitivity than chest X-ray in detecting COVID-19. ${ }^{2}$ Similarly, lung ultrasound findings could prove useful in the follow-up of COVID-19 pneumonia patients.

We conducted this study intending to assess the relationship between lung ultrasound findings and the clinical severity of COVID19 pneumonia. We also sought to compare lung ultrasound with chest X-ray in predicting the clinical severity.

\section{Materials and Methods}

This was a single-center prospective observational study conducted at an academic tertiary care hospital. Institute Ethics
1-3,6-11,13-15 Department of Anesthesia and Intensive Care, Postgraduate Institute of Medical Education and Research, Chandigarh, India

4,5 Department of Radiodiagnosis, Postgraduate Institute of Medical Education and Research, Chandigarh, India

${ }^{12}$ Department of Internal Medicine, Postgraduate Institute of Medical Education and Research, Chandigarh, India

Corresponding Author: Rajarajan Ganesan, Department of Anesthesia and Intensive Care, Postgraduate Institute of Medical Education and Research, Chandigarh, India, Phone: +91 9815930510,e-mail: Raja2n@ gmail.com

How to cite this article: Goel A, Ganesan R, Dogra N, et al. Lung Ultrasound Predicts Clinical Severity of COVID-19 Pneumonia. J Perioper Echocardiogr 2019;7(2):28-32.

Source of support: Nil

Conflict of interest: None

Committee approval was obtained for the study (IEC/INT/2020/ SPL589) and verbal informed consent was obtained from the patients or their relatives. The study was conducted between June 2020 and August 2020 in the dedicated COVID-19 intensive care unit (ICU) and patients were included if they were confirmed COVID-19 positive by Reverse Transcriptase-Polymerase Chain Reaction (RT-PCR). Patients were excluded if they were unable to cooperate with the study.

Data collected included age, sex, comorbidities, respiratory rate, mode of oxygenation, the ratio of the partial pressure of

(0) The Author(s). 2019 Open Access This article is distributed under the terms of the Creative Commons Attribution 4.0 International License (https:// creativecommons.org/licenses/by-nc/4.0/), which permits unrestricted use, distribution, and non-commercial reproduction in any medium, provided you give appropriate credit to the original author(s) and the source, provide a link to the Creative Commons license, and indicate if changes were made. The Creative Commons Public Domain Dedication waiver (http://creativecommons.org/publicdomain/zero/1.0/) applies to the data made available in this article, unless otherwise stated. 
oxygen in arterial blood to the fraction of inspired oxygen (P/F ratio), ICU stay, chest X-ray, point of care ultrasound images, and ICU mortality.

\section{Protocol for Performing Point of Care Ultrasound of Lung}

\section{Equipment}

Sonosite ${ }^{\mathrm{TM}}$ M-Turbo ultrasound system (Sonosite ${ }^{\mathrm{TM}}$ Inc., WA 98201, USA), curved probe (5-2 MHz), and linear probe (13-6 MHz) were chosen according to the body habitus (curved probe was used when subcutaneous tissue and muscles were thicker). The ultrasound probe was cleaned with sterile wipes before and after use. The ultrasound machine was dedicated to COVID-19 positive patients and not moved outside the ICU to avoid cross-contamination.

\section{Procedure}

The ultrasound examination was performed by two physicians with five years of experience each, in performing lung ultrasound examination. A minimum of 4 lung areas, out of the 14 lung areas described by Soldati et al., were scanned during each study. ${ }^{5}$ In the 14 area scan, the mid-clavicular line, mid-axillary line, and internipple line form the landmarks for the anterior and lateral regions and para-vertebral line, shoulder blade's inferior angle, spine, and curtain form the landmarks posteriorly. The video loops of 2 seconds each were stored locally and transferred in video format for analysis offline.

\section{Lung Ultrasound Score}

The video loops were interpreted by an investigator who was blinded to the clinical status of the patient and had 3 years of experience in performing and interpreting lung ultrasound examinations. For each video loop, a score was allotted between 0 and 3 as per the recommendation by Soldati et al. and is briefly as follows (Fig. 1): ${ }^{5}$

Score 0-Pleural line is intact and regular.

Score 1-Pleural line is indented with the area of white below it. Score 2-Pleural line is broken with subpleural consolidation.

Score 3-Extensive white lines in the lungs.

Hence, a score was allotted for each lung area scanned during an ultrasound examination. The median of scores from a lung ultrasound examination was compared with the chest
X-ray and clinical severity at that point of time. A subset of 250 video loops was scored by another investigator, who was also blinded to the clinical status of the patient, to assess the inter-rater agreement.

The chest X-ray from the same day as the lung ultrasound examination was reviewed by a radiologist. The scoring of chest $\mathrm{X}$-ray was performed as described by Borghesi et al. ${ }^{6}$ Briefly, the lung fields were divided into six zones with one line passing along the inferior border of the aortic arch and another passing below the inferior pulmonary vein. The score was given between 0 and 3 for each zone, based on the nature and density of infiltrates. Figure 2 depicts the scoring done on a chest X-ray.

\section{Statistical Analysis}

Statistical analysis was performed using SPSS. Data were described as mean and standard deviation or median and interquartile range. Spearman's rank correlation was obtained between chest $\mathrm{X}$-ray score, median lung ultrasound score, the ratio of the partial pressure of oxygen to fraction of inspired oxygen (P/F ratio), the ratio of pulse oximetry/fraction of inspired oxygen to respiratory rate (ROX index), ${ }^{7}$ Sequential Organ Failure Assessment (SOFA) score, and ICU stay. For patients with multiple studies, the median lung ultrasound score and chest X-ray score obtained in the first study for that particular patient was used for correlation with ICU stay. Mann-Whitney $U$ test was used to compare the chest X-ray score, median lung ultrasound score, $\mathrm{P} / \mathrm{F}$ ratio, respiratory rate, and ROX index between patients who survived and patients who died during their ICU stay. Inter-rater agreement was assessed using Cohen's weighted kappa.

\section{Results}

Bedside lung ultrasound examination was performed in 29 patients (Male:Female-18:11) with a mean age of $54.7 \pm 15.3$ years. Diabetes, hypertension, obesity, hypothyroidism, and chronic obstructive pulmonary disease were present in $8,6,5,2$, and 1 patient respectively. A total of 50 lung ultrasound scans were performed, of which 14 patients had multiple examinations. The total number of images was 438.

The respiratory status of patients and the number of areas scanned at the time of lung ultrasound are shown in Table 1. The P/F ratio, respiratory rate, and ROX index were $154[85,259] \mathrm{mm} \mathrm{Hg}, 24$
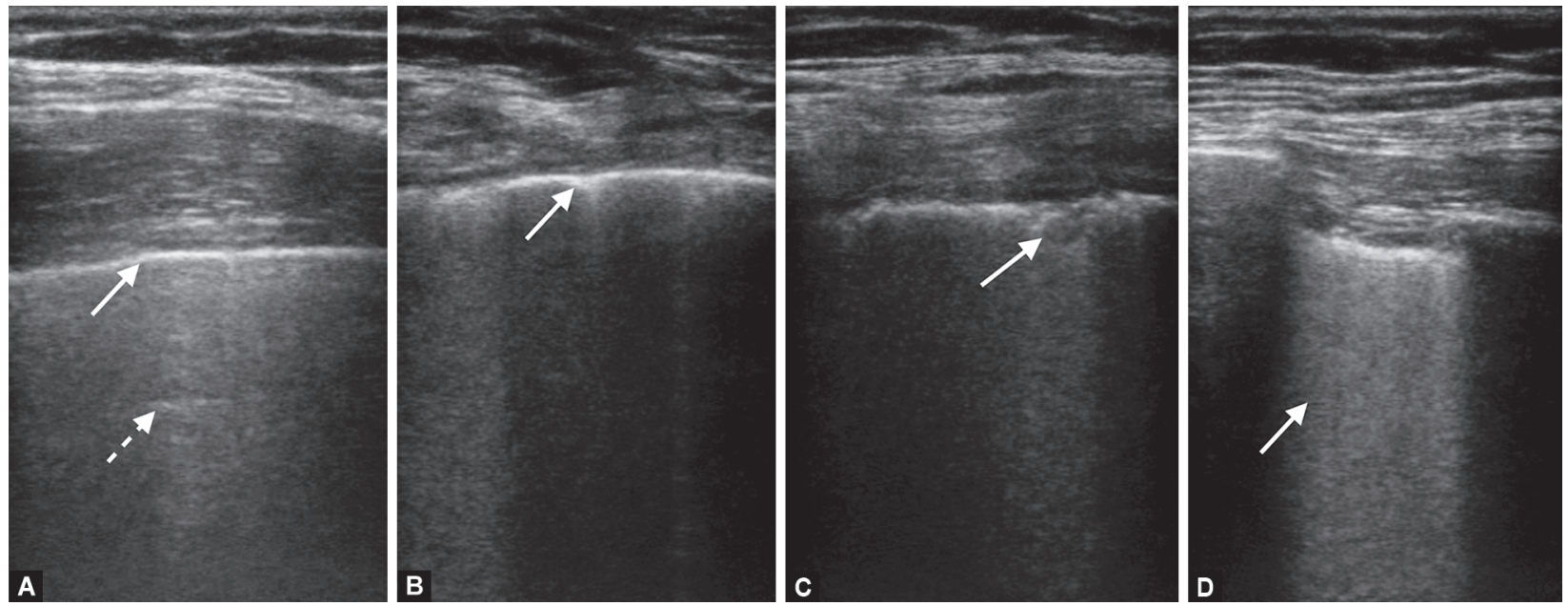

Figs 1A to D: Lung ultrasound scoring done on a set of four images: (A) Score 0: intact pleural line (solid arrow) and A line (dashed arrow); (B) Score 1: indented pleural line (solid arrow); (C) Subpleural consolidation (solid arrow); (D) Extensive white lines beneath the pleura (solid arrow) 


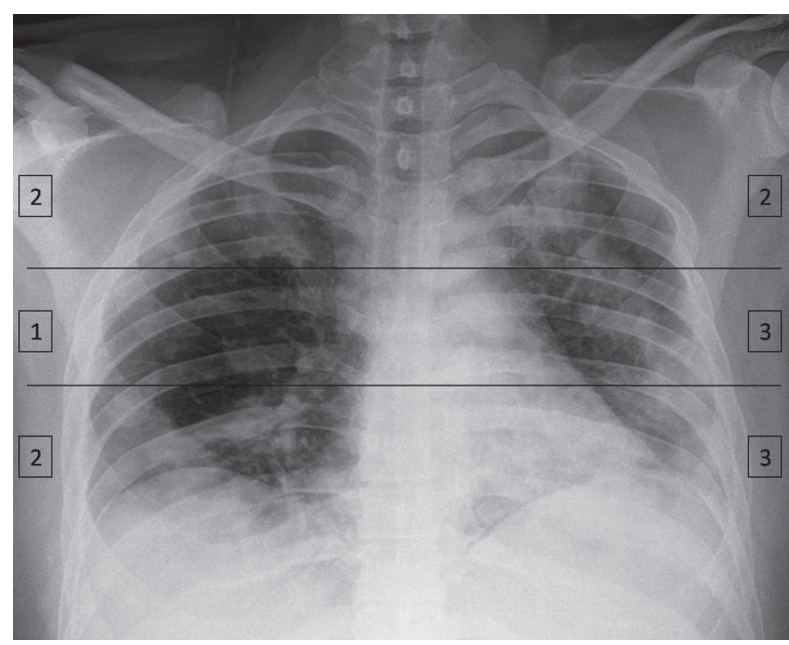

Fig. 2: Chest X-ray scoring done as part of the study

Table 1: Oxygenation status and the number of areas scanned at the time of lung ultrasound examination

\begin{tabular}{llc}
\hline Parameter & Value & $n(\%)$ \\
\hline P/F ratio $(\mathrm{mm} \mathrm{Hg})$ & $>300$ & $10(20)$ \\
& $200-300$ & $8(16)$ \\
& $100-200$ & $16(32)$ \\
& $<100$ & $16(32)$ \\
Oxygen support & Room air & $10(20)$ \\
& Venturi mask & $7(14)$ \\
& High flow nasal oxygen & $27(54)$ \\
& Noninvasive ventilation & $3(6)$ \\
Number of areas scanned & Mechanical ventilation & $3(6)$ \\
& 4 & $11(22)$ \\
& 6 & $6(12)$ \\
& 8 & $11(22)$ \\
& 10 & $8(16)$ \\
& 12 & $3(6)$ \\
& 14 & $11(22)$ \\
\hline
\end{tabular}

$\mathrm{P} / \mathrm{F}$ ratio, Ratio of the partial pressure of oxygen in arterial blood to the fraction of inspired oxygen

$[22,30]$ breaths per minute, and $5.9[4.2,9.5]$, respectively. Among the study patients, two patients on mechanical ventilation during their ICU stay survived and six died. Intact pleural line, indented pleural line, subpleural consolidation, and extensive white lines were seen in 96 (22\%), 42 (9\%), 189 (43\%), and 111 (25\%) of the ultrasound images, respectively.

There was statistically significant correlation between chest $X$-ray score and ROX index (rho $=-0.345, p=0.019$ ), chest X-ray score and ICU stay (rho $=0.434, p=0.049)$, median lung ultrasound score and $\mathrm{P} / \mathrm{F}$ ratio (rho $=-0.543, p<0.001$ ), and median lung ultrasound score and ROX index (rho $=-0.522, p<0.001$ ) (Table 2 and Fig. 3).

There was no statistically significant difference in imaging or clinical parameters between the survivors and non-survivors (Table 3). Using the subset of images scored by the second investigator, Cohen's kappa of 0.601 was obtained for inter-rater agreement (Fig. 4). ${ }^{8}$

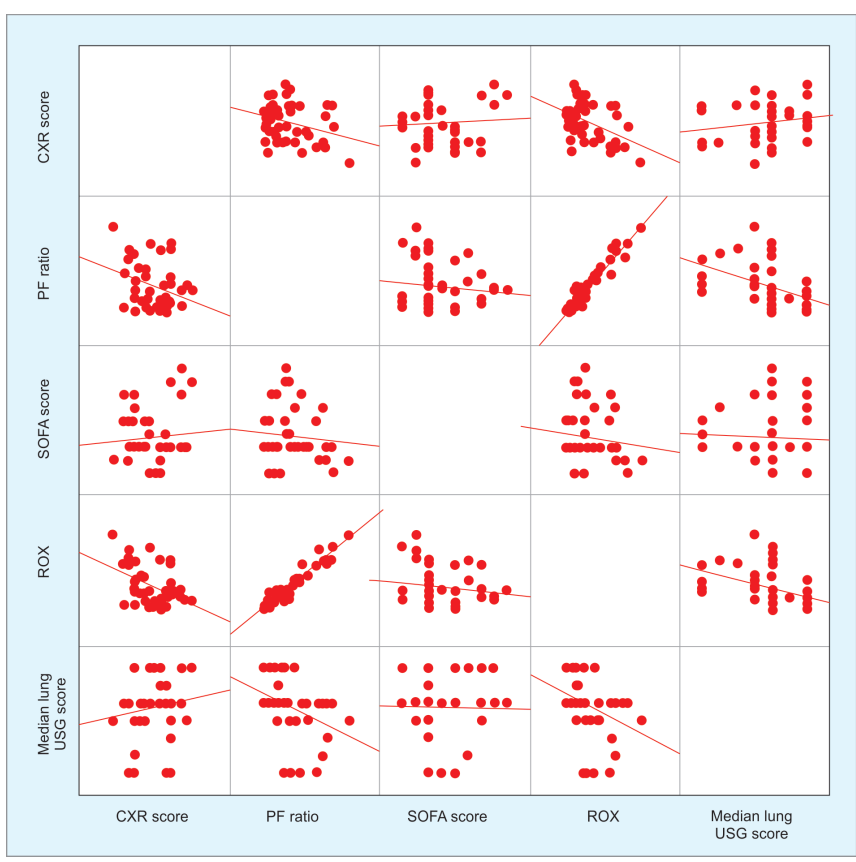

Fig. 3: Spearman correlation between lung imaging features and oxygenation status. CXR score, Chest X-ray score; Lung USG score, Lung ultrasound score; PF ratio, Ratio of the partial pressure of oxygen in arterial blood to the fraction of inspired oxygen; SOFA score, Sequential Organ Failure Assessment score

Table 2: Correlation between lung imaging features, oxygenation status, and intensive care unit (ICU) stay

\begin{tabular}{llcc}
\hline Correlation between & & Spearman's rho & p value \\
\hline Chest X-ray score & P/F ratio & -0.224 & 0.134 \\
& ROX index & -0.345 & $0.019^{*}$ \\
& ICU stay & 0.434 & $0.049^{*}$ \\
Median lung & P/F ratio & -0.543 & $<0.001^{*}$ \\
ultrasound score & & & \\
& ROX index & -0.522 & $0.001^{*}$ \\
& ICU stay & 0.337 & 0.125 \\
& Chest X-ray score & 0.148 & 0.327 \\
\hline
\end{tabular}

P/F ratio: Ratio of the partial pressure of oxygen in arterial blood to the fraction of inspired oxygen

${ }^{*} p$ value $<0.05$, statistically significant

Table 3: Comparison of the lung imaging features and oxygenation status at the time of first lung ultrasound between survivors and nonsurvivors (Mann-Whitney $U$ test)

\begin{tabular}{llll}
\hline Parameters & Survivors & Non-survivors & p value \\
\hline P/F ratio & $108[77,316]$ & $124[68,174]$ & 0.445 \\
ROX index & $5.55[4.21,11.42]$ & $4.07[3.12,5.43]$ & 0.071 \\
Chest X-ray score & $8.5[6,12]$ & $13[6.5,15.5]$ & 0.232 \\
$\begin{array}{l}\text { Median lung } \\
\text { ultrasound score }\end{array}$ & $2[1.5,2]$ & $2[1.87,2]$ & 0.813 \\
\hline
\end{tabular}

P/F ratio: Ratio of partial pressure of oxygen in arterial blood to the fraction of inspired oxygen

\section{Discussion}

Our study suggests that bedside lung ultrasound can be a useful tool for determining the severity of the disease in COVID-19. There 


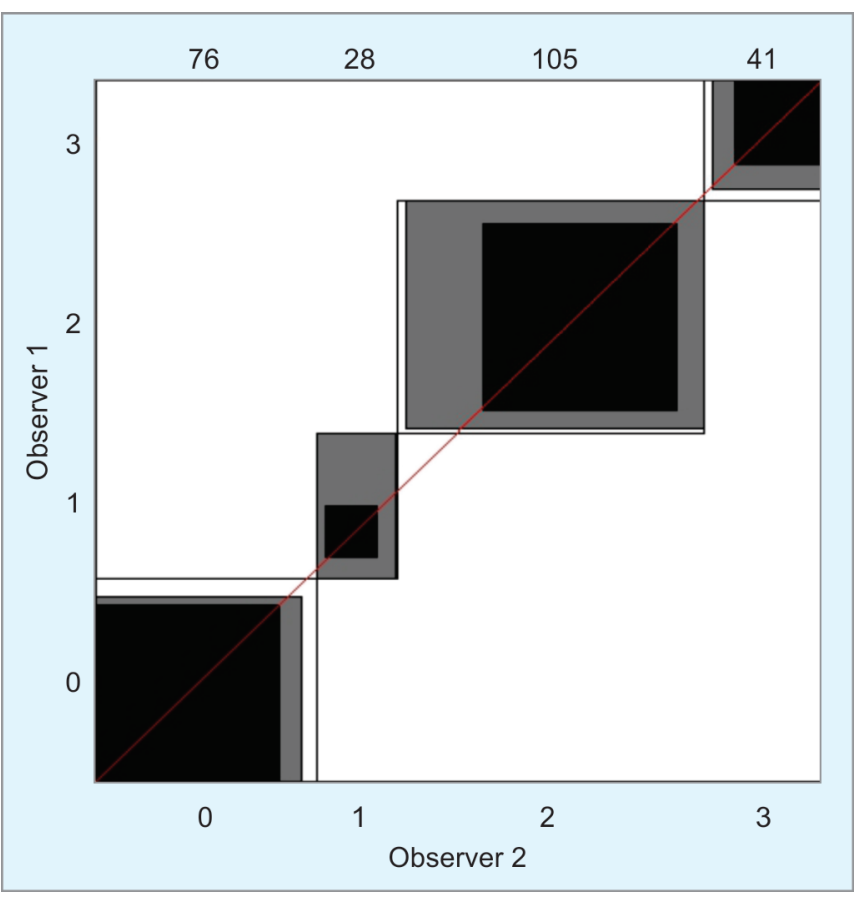

Fig. 4: Agreement chart between two observers on a subset of 250 images. The dimensions of the rectangles represent the number of observations and the shading represents the agreement between the observers with darker shades representing more agreement. ${ }^{8}$ The diagonal line represents the line of no bias

was a moderate correlation of median lung ultrasound score with the lung function as estimated by P/F ratio and ROX index. ${ }^{9}$ Interrater agreement was moderate in scoring the lung ultrasound images, which validates the reliability of lung ultrasound in COVID19 patients. $^{10}$

Our study results are consistent with earlier studies evaluating the relationship between lung ultrasound and clinical severity. In a previous study of lung ultrasound findings in 109 COVID-19 patients, bilateral involvement, pleural thickening, B profile, and confluent $B$ lines were associated with disease severity classified as mild, severe, and critical. ${ }^{11}$ In another study, a higher total lung ultrasound score was associated with increasing severity, with severity graded as mild, moderate, and severe. ${ }^{12}$ When severity was graded according to the $\mathrm{P} / \mathrm{F}$ ratio, Castelao et al. found a correlation between total lung score and $\mathrm{P} / \mathrm{F}$ ratio of $-0.765 .{ }^{13}$ Our study is unique in that we had substantially more number of patients with severe disease $(82.75 \%)$ and we used the ROX index, which better correlates with the severity of respiratory failure. ${ }^{7}$

Lung ultrasound has been shown to outperform chest X-ray in monitoring the respiratory condition of ventilated patients in non-COVID diseases. ${ }^{14}$ In a study of 52 patients with COVID-19, the CURB-65 severity score had a correlation of 0.53 and 0.52 with lung ultrasound score and chest X-ray score, respectively. ${ }^{15}$ On the contrary, we observed only a weak correlation between chest $X$-ray and ROX index, while there was no correlation between chest $\mathrm{X}$-ray score and P/F ratio. Autopsy studies in COVID-19 indicate the possibility of endothelial damage and microthrombi in the pulmonary vasculature. ${ }^{16}$ In a case series by Yusuf et al., areas of subpleural consolidation are avascular and suggestive of infarcts. ${ }^{17}$ Hence, it is possible that the disease severity may be more easily detectable with lung ultrasound than with chest X-ray. The good inter-rater agreement of lung ultrasound images seen in our study has also been demonstrated earlier. ${ }^{18}$

Our study has a few limitations. We did not perform a 14 area lung scan in every patient as we were constrained by the patient position that was mandated by their clinical condition. Our clinical protocol involved proning or lateral decubitus positioning of all patients with respiratory involvement. It is plausible that the severity of hypoxia relates to the amount of lung involved and correlation could improve if all areas are scanned in every patient. Nevertheless, we found a moderate correlation with only the median lung ultrasound score. This suggests the possible clinical utility of a limited lung ultrasound examination protocol in the COVID-19 patients.

To conclude, bedside lung ultrasound examination reflects the clinical severity of COVID-19 pneumonia and has the potential to allow routine monitoring of these patients.

\section{References}

1. Parasher A. COVID-19: current understanding of its pathophysiology, clinical presentation and treatment. Postgrad Med J 2020. DOI: 10.1136/postgradmedj-2020-138577.

2. Lomoro P, Verde F, Zerboni F, et al. COVID-19 pneumonia manifestations at the admission on chest ultrasound, radiographs, and $\mathrm{CT}$ : single-center study and comprehensive radiologic literature review. Eur J Radiol Open 2020;7:100231. DOI: 10.1016/j. ejro.2020.100231.

3. Lopes AJ, Mafort TT, Costa $\mathrm{CH}$, et al. Comparison between lung ultrasound and computed tomographic findings in patients with COVID-19 pneumonia. J Ultrasound Med 2020. DOI: 10.1002/ jum.15521.

4. Convissar DL, Gibson LE, Berra L, et al. Application of lung ultrasound during the COVID-19 pandemic: a narrative review. Anesth Analg 2020;131(2):345-350. DOI: 10.1213/ANE.0000000000004929.

5. Soldati G, Smargiassi A, Inchingolo R, et al. Proposal for international standardization of the use of lung ultrasound for patients with COVID-19: a simple, quantitative, reproducible method. J Ultrasound Med 2020;39(7):1413-1419. DOI: 10.1002/jum. 15285.

6. Borghesi A, Zigliani A, Masciullo R, et al. Radiographic severity index in COVID-19 pneumonia: relationship to age and sex in 783 Italian patients. Radiol Med 2020;125(5):461-464. DOI: 10.1007/s11547-02001202-1.

7. Roca O, Messika J, Caralt B, et al. Predicting success of high-flow nasal cannula in pneumonia patients with hypoxemic respiratory failure: the utility of the ROX index. J Crit Care 2016;35:200-205. DOI: 10.1016/j. jcrc.2016.05.022.

8. Bangdiwala SI, Shankar V. The agreement chart. BMC Med Res Methodol 2013;13(1):97. Available from: http://dx.doi. org/10.1186/1471-2288-13-97.

9. Schober P, Boer C, Schwarte LA. Correlation coefficients: appropriate use and interpretation. Anesth Analg 2018;126(5):1763-1768. DOI: 10.1213/ANE.0000000000002864.

10. McHugh ML. Interrater reliability: the kappa statistic. Biochem Med 2012;22(3):276-282. DOI: 10.11613/bm.2012.031.

11. Sahu AK, Mathew R, Bhoi $S$, et al. Lung sonographic findings in COVID-19 patients. Am J Emerg Med 2020. DOI: 10.1016/j.ajem.2020. 08.080 .

12. Lichter $Y$, Topilsky $Y$, Taieb $P$, et al. Correction to: lung ultrasound predicts clinical course and outcomes in COVID-19 patients. Intensive Care Med 2020;46(11):2128-2129. DOI: 10.1007/s00134-02006254-5.

13. Castelao J, Graziani D, Soriano JB, et al. Findings and prognostic value of lung ultrasound in COVID-19 pneumonia. J Ultrasound Med 2020. Online ahead of print. Available from: http://dx.doi.org/10.1002/ jum.15508. 
14. Lichtenstein D, Goldstein I, Mourgeon E, et al. Comparative diagnostic performances of auscultation, chest radiography, and lung ultrasonography in acute respiratory distress syndrome. Anesthesiology 2004;100(1):9-15. DOI: 10.1097/00000542-20040100000006.

15. Bhandari S, Singh A, Bagarhatta $M$, et al. Evaluation of clinicoradiological profile and correlation with ultrasonography of the chest in coronavirus disease 2019 pneumonia. Indian J Med Specialit 2020;11(2):70. DOI: 10.4103/injms.injms_55_20.
16. Maiese A, Manetti AC, La Russa R, et al. Autopsy findings in COVID19-related deaths: a literature review. Forensic Sci Med Pathol 2020. 1-18. DOI: 10.1007/s12024-020-00310-8.

17. Yusuf GT, Wong A, Rao D, et al. The use of contrast-enhanced ultrasound in COVID-19 lung imaging. J Ultrasound 2020. 1-5. DOI: 10.1007/s40477-020-00517-z.

18. Kumar A, Weng Y, Graglia S, et al. Interobserver agreement of lung ultrasound findings of COVID-19 10.1101/2020.08.16. 20176156. 